\title{
L'agent glottopolitique et son glossaire Enjeux de représentations des langues chez trois romanciers hétérolingues
}

\author{
Diane Schwob \\ Laboratoire Ligérien de Linguistique, UMR 7270 CNRS, Université d'Orléans, France \\ diane.schwob@univ-orleans.fr
}

\begin{abstract}
Résumé. Quand dire, c'est feindre...L'analyse des emprunts aux langues endogènes dans les romans hétérolingues génère une interrogation sur leur dimension mimétique. Si la critique linguistique et la sociocritique des littératures francophones tendent à percevoir les romans hétérolingues comme représentatifs des contextes plurilingues, en revanche les partisans de l'hétérolinguisme mettent en évidence la dimension illusoire de cette représentation. Quand dire, c'est faire...En revanche, le glossaire des écrivains hétérolingues tend à imposer en langue les emprunts, dévoilant ainsi sa portée glottopolitique. Les pratiques glossairistiques spécifiques des écrivains peuvent ainsi être mises en relation avec leurs représentations épilinguistiques déclarées, comme avec celles des locuteurs endogènes de la société dont ils émanent. L'analyse de trois romans francophones (Le Jujubier du Patriarche, d'Aminata Sow Fall, 1993, Allah n'est pas obligé, de Kourouma, 2000, et Le Rocher de Tanios, de Maalouf, 1993) révèle l'intérêt des comparaisons du glossaire des écrivains pour découvrir plus en profondeur la spécificité de leur rapport à la langue littéraire.
\end{abstract}

\begin{abstract}
The glottopolitical agent and his glossary. Languages representation issues in three heterolingual novels. How to dissemble things with words...The analysis of the elements borrowed from endogenous languages questions their mimetic dimension. Even if, on one hand, linguistic criticism and sociocriticism of french-speaking literature tend to consider heterolingual novels as representations of plurilingual contexts, on the other hand, supporters of heterolinguism underline the illusory aspect of such representations : How to do things with words...But glossaries put together by heterolinguistic writers in their works tend to impose the elements borrowed upon the language used, thus asserting its glottopolitical scope. Glossaristic practices specific to the writers can be put into relation with their asserted epilinguistical representations, as well as with the endogenous speakers' who belong to the same society. The analysis of three
\end{abstract}


french-speaking novels (Sow Fall's "Jujubier du patriarche", Kourouma's "Allah n'est pas obligé" and Maalouf's "Le Rocher de Tanios") stresses the importance of comparating the writers' glossaries to have a better understanding of the specifics of their relationships with the literary language they use.

Cet article s'inscrit dans l'axe francophone du colloque et abordera la question de la représentation des langues endogènes dans trois romans hétérolingues en la confrontant aux pratiques glossairistiques de leurs auteurs. Dans le cadre d'une norme pluricentrique (Pöll 2005 : 17-28), les écrivains hétérolingues textualisent dans leurs œuvres le contact des langues, «idiomes étrangers [ou] variétés (sociales, régionales, chronologiques) de la langue auctoriale » (Grutman 1996 : 72). C'est ainsi que le français de référence se mêle : au wolof, dans Le Jujubier du patriarche (1993) de Sow Fall, sénégalaise ; au malinké, dans Allah n'est pas obligé (2000) de Kourouma, ivoirien ; au syro-libanais dans Le Rocher de Tanios (1993), de Maalouf, franco-libanais. Cette textualisation peut s'analyser dans le cadre d'une linguistique variationniste, comme un continuum sur un axe marqué par six échelons (Grutman 2002 : 335), du degré minimal - où L1, langue principale de l'écriture, commente L2 sans la citer - jusqu'au code-switching. Pris sur cet axe, l'usage des emprunts est commun à nos trois auteurs ; parmi eux, les emprunts (Deroy 1956 : 224) lexématiques, issus des langues endogènes, restent en quête d'intégration phonique, phonologique et graphique (Queffélec 1998 : 246-248). L'ensemble de ces emprunts et de leurs gloses crée dans les romans un glossaire, dont nous avons proposé une méthode d'analyse (Schwob 2014) inspirée du modèle conçu par Thibault (2006) pour une glossairistique francophone.

Il s'agit à présent de mettre en perspective cette méthode, en situant son objet au sein des champs théoriques qui en pratiquent l'étude. Qu'est-ce que l'examen d'un tel glossaire peut apporter aux descriptions sémiotiques et lexicographiques des œuvres hétérolingues ? Pour le savoir, nous chercherons, par le prisme de la mise en scène des langues endogènes dans la pratique de l'emprunt, « comment [...] le texte du roman parle la langue » (Gauvin 1999 : 54) : le roman hétérolingue peut-il être considéré comme représentatif du contact des langues dans une situation sociolinguistique donnée - la nomenclature des emprunts de l'œuvre, comme une sélection fidèle des langues endogènes, substrat linguistique du roman ? Ou bien ce glossaire est-il l'indice, par la méthode qu'il engage, d'un positionnement esthétique et sociolinguistique auctorial ? Comment alors, appréhender la mise en scène des vernaculaires dans le roman, et que nous apprend-t-elle de l'imaginaire des langues chez les auteurs ? Bref, avec la transmutation opérée par le texte littéraire, comment appréhender sa langue, du « fait de langue » à «l'effet de langue » (op. cit. : 54) ? Nous évoquerons donc les enjeux de représentation des langues selon deux acceptions du terme. Prenant d'abord le mot en son sens d'imitation, nous soulèverons la question du réalisme linguistique en interrogeant une éventuelle mimèsis des langues endogènes orchestrée par le glossaire romanesque. Mais en textualisant le contact des langues, le roman hétérolingue non seulement donne à lire des lexies endogènes, mais reproduit aussi une situation sociolinguistique donnée, tentant ainsi, « en français, de rendre compte du répertoire en jeu dans l'échange des locuteurs, qui manient plusieurs codes linguistiques, selon les fonctions dévolues à leur usage » (Prignitz 2004 : 26). Or, cette mise en scène créatrice, représentation des langues en contact, est toujours aussi une stylisation qui engage le regard de l'écrivain, ses représentations sur les langues, au sens de 
« formes de connaissances socialement élaborées » (Blanchet $2007: 272$ ) accessibles via les discours épilinguistiques. De fait, les sociolinguistes s'accordent à penser que «les langues sont des abstractions construites à partir d'une certaine compréhension de certaines pratiques et de certaines représentations [...], et non des "objets réels" qui s'imposeraient à nous comme des "données" », d'où « l'importance accordée aux "représentations" » (op. cit. : 261262). Dans un deuxième temps, nous envisagerons donc l'emprunt et son traitement dans le cadre du paradigme interprétatif reliant les pratiques aux représentations.

\section{Fait ou effet ? L'emprunt littéraire à la croisée des champs théoriques}

\section{1 «Quand dire, c'est feindre... ». Enjeux mimétiques de la représentation des langues}

«Quand dire, c'est feindre... » (Fonseca 1996 : 883) : quelle mimèsis du contact des langues dans les romans du corpus? Une partie de la critique littéraire francophone aborde les œuvres via un prisme linguistique ou sociolinguistique, voire sociologique et transculturel : « laboratoire social avancé de l'interculturel, le discours romanesque [francophone] devrait nous montrer comment coexistent les langages issus d'époques et de périodes différentes qui jalonnent l'expérience du vécu sociopolitique et culturel ». Ce postulat d'un «mimétisme discursif » (Barry 2007 : 20-23) du roman, «phénomène pluristylistique, plurilingual, plurivocal » s'autorise notamment de la polyphonie bakhtinienne (1978).

Issus de la traductologie et du comparatisme, les tenants de l'hétérolinguisme opposent au postulat mimétique le postulat structuraliste de l'autonomie du texte littéraire. En effet, si les contextes sociolinguistiques peuvent en partie inspirer leur représentation textuelle, transposer la notion de diglossie en littérature risque de réduire celle-ci à une simple «"chambre d'enregistrement" d'une situation définie au plan macro-social» (Gauvin 2001 : 157, citant Beniamino). Or, le réalisme linguistique ne saurait redoubler l'exhaustivité du réel, l'emprunt entrant plutôt en rapport synecdochique par rapport au tout du discours social qu'il représente, «à l'aide de quelques touches soigneusement apportées » (Grutman 1997 : 4243). La scène de la diégèse des trois romans étant en Afrique subsaharienne ou dans le Mashrek, le choix de la langue française par nos trois auteurs interroge sur la représentation des vernaculaires par l'emprunt : «la fiction sociolinguistique dans le roman est de faire tenir en français aux personnages leurs discours » (Prignitz 2004 : 31). L'usage de l'emprunt viserait donc à faire entendre les langues endogènes à travers le français. L'approche hétérolinguiste de la textualisation du contact des langues dans le roman emprunte alors à l'analyse de discours les outils nécessaires à la description de cette scénographie par laquelle la parole littéraire convoque une scène d'énonciation. Dans cette stratégie d'écriture, les emprunts, «qui participent à la fois du monde représenté par l'œuvre et de la situation à travers laquelle s'institue l'auteur qui construit ce monde » font office d'«embrayage paratopique » (Maingueneau 2004 : 96-97), signalant de façon stylisée le bruissement des langues endogènes sous la mise en scène fictionalisée des discours sociaux. Mais si, concernant la langue des personnages, fiction d'oralité il y a, quid de la langue du narrateur : est-elle supposée reproduite dans une certaine fidélité sociolinguistique, ou l'imitation se limite-t-elle au niveau des discours directs des personnages sans investir la structure 
énonciative globale du roman? Se pose ainsi la question du «cloisonnement des voix » (Meizoz 2001 : 23). Par conséquent, l'analyse de la visée mimétique de l'emprunt dans les romans interroge non seulement les rapports entre langue commune et langue littéraire, mais aussi les rapports entre oral et écrit en littérature, engageant de façon décisive une esthétique romanesque.

D'ailleurs, sociolinguistes et historiens de la littérature s'accordent à trouver dans cette stylisation même des indices sur le rapport au langage des écrivains, conformément à la revalorisation aristotélicienne de la mimèsis, qui, de simple copie du réel dénoncée par Platon, devient une représentation de celui-ci qui le met en forme, véritable mode de connaissance esthétique du monde. Ainsi, la représentation du contact des langues par les auteurs hétérolingues est susceptible de nous informer sur leurs représentations épilinguistiques. Dufour s'applique à mettre en évidence une histoire de la «pensée romanesque du langage », où le dialogue, «forme pensante », «parle de la parole » (2004 : 302). Pour Favart, la mimèsis du vernaculaire par les écrivains, cet « artefact à deux faces » qui reflète à la fois les représentations du sociolecte chez l'écrivain et chez son lecteur, véhicule des stéréotypes sociaux, «vision linguistique admise par la communauté », constituée à partir de "phénomènes qui, dans la langue parlée, sont communs à la majorité des locuteurs ». A ce titre, elle est donc aussi « un acteur de la variation sociale, car elle la donne à voir, même si cette représentation est fictionnelle » (2009 : 147-148). Ainsi, dans le roman, dire, c'est feindre, dans une certaine mesure. Mais la représentation de la variation a aussi force de légitimation; partant, valeur performative. Comme la situation de diglossie génère chez l'auteur francophone une «surconscience linguistique, [...] une conscience de la langue qui devient à la fois un objet de discours et de métadiscours, un lieu de réflexion privilégié sur le rôle et la nature de la chose littéraire », alors écrire devient un "véritable "acte de langage" », engageant autant «le statut d'une littérature et sa place sur l'échiquier mondial que les modalités d'écriture [ou] poétiques individuelles » (Gauvin 2008 : 15). De ce point de vue, il devient patent que dans le roman, dire... c'est aussi faire.

\section{2 «Quand dire c'est faire ». Enjeux glottopolitiques des représentations sur les langues}

Le roman générerait une certaine illusion du vernaculaire grâce à l'emprunt, connotateur de mimèsis linguistique. En imposant celui-ci du discours à la langue du texte littéraire, il pourrait également porter une action sur le langage. Si la glottopolitique se définit comme la «gestion des pratiques langagières »(Guespin 1985 : 23), les «approches qu'une société a de l'action sur le langage, qu'elle en soit ou non consciente », émanant d'acteurs privés ou publics, du cercle familial ou des sphères institutionnelles; et dans la mesure où le patrimoine littéraire «façonne les imaginaires linguistiques et influence les productions langagières »; alors, « la littérature est [...] une force glottopolitique » dont les écrivains sont les «agents » (Caitucoli 2004a : 2-3), susceptibles de légitimer une langue minorée. Autant dire qu'à côté du postulat d'autonomie, on gardera à l'esprit le postulat de «productivité » du texte littéraire, qui génère une diachronie textuelle susceptible d'entraîner des « modifications de système » (Arrivé 1969 : 12) propres à faire glisser l'emprunt du glossaire fictionnel des écrivains vers l'inventaire lexicographique. 
Or, à nos yeux, le point de vue des lexicographes sur la textualisation du contact des langues dans les littératures francophones autorise un dépassement des antinomies où nous plaçait la question de la mimèsis des langues dans le roman. Wissner (2010 : 38-40) le rappelle, « le dépouillement de corpus littéraires est [...] une approche validée en lexicographie » générale et différentielle : ce type de textes a l'avantage d'être contextualisé par "un maximum de paramètres fiables » sur «son époque, son genre discursif avec ses normes propres, son énonciateur et même le public visé », sans compter « un riche paradiscours ». De ce fait, s'il est «analysé de façon adéquate et comparé à des données fiables », l'usage qu'il consacre peut être considéré comme indicateur non seulement «des jugements linguistiques de la communauté (socio)linguistique à laquelle appartient l'écrivain », mais aussi « des usages de cette dernière [...], et des normes propres au discours concerné, ici littéraire romanesque ».

La lexicographie interprétative de Frey, inspirée de l'approche écologique qui prend en compte « les rapports entre les langues et leur milieu, c'est-à-dire d'abord les rapports entre les langues elles-mêmes, puis entre ces langues et la société » (Calvet 1999 : 17), en fondant son modèle d'analyse sur «le couple pratiques et représentations en étroite relation avec le milieu» (op. cit. : 53), apporte des principes méthodologiques utiles à l'analyse de la textualisation du contact des langues dans le roman. Frey oppose les stratégies d'assimilation de l'emprunt - le locuteur s'approprie la variante « en l'intégrant sans aucune mention dans son discours », en usage ; et de distanciation - le locuteur traite le mot en mention, par des signes de mise à distance et une éventuelle glose (2008 : 30). Ce faisant, Frey prend en considération non seulement les pratiques linguistiques des locuteurs, mais la manière dont leurs représentations sur ces pratiques se manifestent à travers des stratégies énonciatives. Focalisées sur la représentation d'une situation sociolinguistique, de dynamiques langagières, ces analyses permettent ainsi de corréler divers types de traitements de l'emprunt à un positionnement du locuteur. Cette attention au traitement de l'emprunt est perceptible chez Latin, qui développe l'intuition d'une étude de la glossairistique des écrivains d'Afrique subsaharienne, relevant dans Wangrin de Hampâthé Bâ, un «petit lexique » de « 228 notes métalinguistiques». Pour elle, «chaque écrivain développe son système de variation métalinguistique en référence à sa double perception des normes africaine et française et, s'il $\mathrm{y}$ a des différences d'un auteur à l'autre, il y a toujours cohérence interne dans le texte littéraire » (2006 : 148). Ce postulat de tendances convergentes pour l'établissement de profils glossairistiques d'écrivains révélateurs de visées mimétiques et glottopolitiques distinctes semble fécond. Latin propose d'analyser ainsi le positionnement de ce «jury légitime du réalisme linguistique en voie d'émergence » composé d'écrivains de la nouvelle génération africaine francophone : pourquoi ne pas «décrire ce vocabulaire en tenant compte de la première attestation littéraire des termes par ailleurs inventoriés dans l'IFA [afin de] les évaluer en fonction de la position d'énonciation sociolinguistique exacte à laquelle se place l'instance écrivante »? (op. cit. : 148-9). Cette confrontation des diachronies, celle des usages répertoriés par les inventaires de particularités lexicales du français et celle des emprunts utilisés par les écrivains, ouvre des perspectives pour appréhender les visées mimétique et glottopolitique des écrivains glossairistes.

\section{Faits et effets des glossaires : quelle méthode d'analyse ?}

En les infléchissant quelque peu en vue de leur adaptation à notre corpus, mais tout en en suivant l'esprit, nous nous proposons d'explorer ces pistes d'analyses. Qu'elle soit ou non 
associée à un traitement typographique qui les mette en relief, la glose des emprunts constitue souvent une stratégie d'explicitation de leur sémantisme assurant l'intercompréhension entre auteur et lecteur, et manifeste une attention soutenue aux conditions de production et de réception de la langue de l'œuvre envisagée comme discours (Maingueneau 1993). Comme elle met en jeu deux codes linguistiques, nous la décrirons à partir des modes de communication exolingue et endolingue en situation de contact des langues définis par la linguistique interactionnelle. Le premier mode, relevant d'une conception dialogique du discours, entraîne «des ajustements réciproques» des interlocuteurs, alors que dans le second, "les divergences codiques » n'étant «plus perçues comme significatives », ne suscitent pas de reformulations (De Pietro 1988: 71). Nous distinguerons donc trois traitements de l'emprunt, en prenant pour point de référence la langue dont il est issu : traitement endoréférentiel - le lecteur privilégié comprend l'emprunt -, exoréférentiel - le lecteur privilégié ne le connaît pas -, ou polyréférentiel. Pour décrire le traitement exoréférentiel, prise en charge maximale du narrataire exogène, nous nous référons aux articles de lexicographie différentielle, qui constituent un modèle de glossaire philologique pour l'édition des textes hétérolingues. Dans son article «Glossairistique et littérature francophone » (2006), Thibault analyse les glossaires ajoutés à des textes francophones par les éditeurs, traducteurs ou écrivains, qui, sans être lexicographes de profession, souhaitent faciliter l'accès de ces œuvres aux lecteurs exogènes. Sous sa forme «maximal[e] », un glossaire littéraire francophone comportera ces rubriques : entrée, catégorie grammaticale, acceptions, fonctionnement syntagmatique, contexte d'apparition, autres contextes, rubrique encyclopédique, remarques formelles, remarques différentielles, commentaire historicocomparatif, bilan bibliographique.

Inspirée par ces principes, la comparaison des pratiques glossairistiques de nos trois auteurs s'appuie sur des analyses statistiques sur les emprunts lexématiques du corpus, concernant 90 occurrences relevées dans chaque roman, soit 270 occurrences correspondant à 97 lexies différentes - 23 chez Kourouma, 33 chez Maalouf, 35 chez Sow Fall. Ces premières analyses quantitatives (Schwob 2014) mettront en perspective les nouvelles études de cas ci-dessous visant à évaluer les fonctions mimétique et glottopolitique des emprunts. Et pour confirmer ces nouvelles analyses plus qualitatives, nous étendrons notre champ d'étude à d'autres formes, les emprunts sémantiques. Puis, dans un mouvement d'élargissement progressif des cadres d'analyse, nous confronterons les tendances ainsi dégagées aux attitudes des auteurs concernant la langue d'écriture, sollicitant pour ce faire leurs métadiscours sur leurs productions littéraires, et la prise en compte de leurs trajectoires d'écrivains. Enfin, dans une perspective d'analyse écologique de leur inscription dans les champs linguistique et littéraire, nous confronterons leur positionnement glottopolitique aux «cent portraits linguistiques » (Gueunier 1993) des enquêtes sociolinguistiques menées auprès des locuteurs endogènes. Ainsi, la confrontation des pratiques glossairistiques des écrivains, d'une part aux usages endogènes configurant les emprunts considérés, d'autre part à la multiplicité des positionnements de ces locuteurs face aux langues, contribuera à cerner la spécificité de leurs stratégies auctoriales. 


\section{Trois glossaires : trois profils glottopolitiques?}

\subsection{Amin Maalouf : pratique inclusive du glossaire}

Pays phare de la Francophonie, le Liban a toujours « entretenu une relation particulière avec les cultures et les langues grâce à sa situation géographique à la frontière de l'Orient et de l'Occident» (Kanaan 2011: 27). Les romans de Maalouf se caractérisent par une riche variation diatopique, liée au « substrat linguistique de l'Orient musulman (l'arabe, le persan, le turc), et de l'Orient chrétien (l'araméen, l'arménien, le grec et le syriaque) » (Dakroub $2011: 261-263)$.

Dans Le Rocher de Tanios, dont la diégèse se situe au XIX ${ }^{\mathrm{e}}$ siècle, le dialecte vernaculaire du Mont Liban constitue à lui seul une " "synthèse" linguistique, un processus de créolisation continu »(Dakroub, id.). La variation diatopique se croise avec la variation diachronique, témoins des archaïsmes liés au champ vestimentaire du XIX ${ }^{\mathrm{e}}$ siècle, telle la abaya, arborée " par les notables du pays », ou le tantour, coiffe des "dames de la haute société de jadis » (Assaad 2013 : 3), ainsi que le toponyme Roumieh, qui connote un regard oriental sur la ville sainte, usité dans «le parler des maronites du mont Liban au XIX ${ }^{\mathrm{e}}$ siècle » (Dakroub 2014). Bien des mots du parler de Kfaryabda, vernaculaire des montagnards libanais, relèvent des substrats linguistiques pré-islamiques de l'Orient chrétien et byzantin - ainsi les termes syriaques khouriyyé, mar, ataba, bouna, et kfaryabda lui-même. Bref, dans cette œuvre, c'est «tout l'"Orient" qui semble parler», soit «horizontalement, à travers ses régions géographiques », soit «verticalement à travers ses époques historiques » (Dakroub 2011 : 263-264).

Cette scrupuleuse visée mimétique passant par l'emprunt incite à interroger les choix d'écriture du roman en français plutôt qu'en arabe. La biographie langagière de Maalouf fait état d'une nette partition des fonctions des langues, entre arabe, «langue sociale, celle dans laquelle je m'exprimais en public, oralement comme par écrit », et français, langue de la découverte du «monde, [des] idées, [de] la littérature » (citation Volterrani 2001). Son choix du français est relié à une paradoxale visée d'exactitude mimétique : «lorsque j'ai essayé d'écrire des textes de fiction en arabe, j'ai été gêné par la différence qui existe entre la langue parlée et la langue écrite »; dans un «texte de fiction et surtout dans un dialogue, je n'ai jamais pu dépasser la barrière psychologique qui consiste à faire parler un personnage dans une langue que personne ne parle actuellement» (citation Solon 2004 : 173). C'est pour transposer fidèlement la langue orale dans l'écrit du roman que Maalouf a choisi le français, plus propice à la variation que l'arabe classique. Les personnages sont donc censés parler leurs langues, dont les emprunts sont les embrayeurs, à côté d'un narrateur "initial bilingue et francophone qui "rapporte" les "faits" de paroles des autres personnages en les "transposant" ou en les traduisant [en] français » (Dakroub 2011 : 266)... Alami note le paradoxe de cette transposition en langue étrangère, dans «le roman du local par excellence », de la parole, «non [de] personnages qui tendent vers l'universalité comme c'est le cas dans les autres romans», mais de «montagnards libanais aux antipodes de tout universalisme » $(2005: 33)$. 
La mise en scène de la variation diatopique est poussée jusqu'en ses dimensions phonologiques, notées dans la graphie, ainsi la «prononciation du mot khwéja (Le Rocher de Tanios) qui devient khwajé (Samarcande), parce que dans le premier, c'est la variante phonétique du mot turcopersan khwajé, telle qu'elle est utilisée par les villageois [...] du Mont Liban » (Dakroub 2011 : 272).

Sur le plan sémantique, s'observent des métacalques, comme dans ce passage : «eux aussi avaient dû promettre, la main sur le duvet de la moustache » (Maalouf 1993 : 43), légèrement modulé par l'ajout maaloufien du « duvet » qui connote la jeunesse des protagonistes. Certes, « un lecteur arabophone distinguera la formule originale ("la main sur la moustache") de la formule remaniée par Amin Maalouf» (Assaad 2004 : 475), et de même dans les imprécations adressées par les Montagnards à la Cheikha, « outre de lait tourné », « femmeronce née des lunes du Jord» (Maalouf 1993 : 47). Dans une enquête sollicitant le sentiment linguistique de locuteurs libanais âgés, Assaad découvre que " la première chaîne syntagmatique relève d'un "discours répété" », appartenant au « registre des calques d'expression à sens métaphorique », alors que « la seconde, qui lui est juxtaposée, ne possède aucun équivalent arabe ». En revanche, «les Français interviewés sont restés perplexes », concluant unanimement que ces expressions «sont imagées, exotiques et évocatrices d'un folklore local » $(2013: 7)$. La mimésis linguistique maaloufienne fait donc entendre à la fois, dans la phrase française, la précision d'une variation diatopique et diachronique panorientale, et l'audace des défigements, notamment dans le cas des métacalques : Assaad décrit ces pratiques d'écriture comme une «mutation linguistique ». Pour l'auteur (cité par Assaad 2004 : 471), « chacune des deux langues influe sur l'autre » : ni le français, ni l'arabe dialectal, ne sortent intacts de cette alchimie.

La nomenclature des emprunts lexématiques, et cela est confirmé par les calques sémantiques, orchestre donc une mimésis des parlers vernaculaires, mais organise aussi la poiesis du roman. Une longue glose permet de faire connaître au lecteur le système des anthroponymes du village, fondé sur une opposition entre les noms villageois pris aux saints du calendrier, dont celui du personnage éponyme - Tanios, alors précédé de l'archaïsme mar - et les noms plus flamboyants de la lignée du Cheikh, «Sakhr, Raad, Hosn, qui signifient "rocher", "tonnerre", "forteresse" ». L'évocation de ces «coutumes précises en matière de prénoms » convoque la langue montagnarde en ce qu'elle a de plus idiosyncrasique tout en compliquant la quête des origines du personnage, pivot du roman (Maalouf 1993 : 47-50). Avec la même précision philologique, Maalouf décline son propre patronyme selon de multiples graphies dans Origines, faisant à la fois entendre symboliquement la langue dans sa variation diachronique et diatopique, et compliquant singulièrement, motif prégnant de son œuvre, la quête des origines par le chemin de la langue. Ainsi le traitement de la nomenclature de ses emprunts manifeste le croisement des visées mimétique et poétique.

Que révèle le glossaire maaloufien sur le plan glottopolitique? Dans Origines toujours, Maalouf évoque la graphie du nom de son village : «Kfar-Yaqda, altéré dans le parler local en Kfar-Ya'da par adoucissement du $q$ guttural sémitique, et que j'ai parfois transformé en Kfaryabda, croyant ainsi le rendre plus prononçable » (2004). Cette confidence suggère un équilibrage de la mimèsis des langues endogènes par un positionnement glottopolitique spécifique, orienté vers le lecteur exogène. Il est cohérent avec le traitement plutôt exoréférentiel des emprunts, que révèle l'analyse quantitative du Rocher de Tanios. La mise à 
distance typographique par les italiques, opposant les emprunts à la norme du français de référence, s'adresse plutôt au lecteur exogène, auquel la glose interlinéaire, intégrée au fil de la diégèse sans insertion de notes, évite l'effort d'interrompre sa lecture pour la consulter. De nos trois auteurs, c'est Malouf qui pratique la glose la plus longue, 4 lignes en moyenne par emprunt, avec souvent une dimension encyclopédique, notamment pour oubour, 18 lignes (Maalouf 1993 : 43), kichk, 24 1. (op. cit. : 74), et kaff, 49 1. (op. cit .: 18), avec en plus une perspective différentielle distinguant le fonctionnement sémantique des termes libanais de celui de leur traduction française - passage, pois chiche et main : ces pratiques donnent au narrateur un éthos de philologue. Son glossaire, le plus proche du programme de Thibault, génère un accompagnement didactique du lecteur exogène, dont les lacunes lexicales et culturelles sont palliées par ces rubriques substantielles. L'écrivain affirme une orientation universaliste cohérente avec ce glossaire exoréférentiel, le livre n'étant pas conçu « pour des Français ou bien pour des Libanais uniquement », mais s'adressant «au lecteur en général » (citation Assaad $2013: 8$ ).

Mais à côté de ce positionnement glottopolitique visant un élargissement du champ littéraire francophone par la prise en compte des compétences du lecteur exogène, la présence des calques et la multiplicité des emprunts dans son œuvre suggère une imposition dans le français des langues endogènes convoquées par la diégèse. Interrogé sur les calques qu'il ne glose que rarement, l'auteur affirme qu'il «n'a pas toujours voulu présenter » ces expressions en les «soulignant clairement », «parce qu'il a "senti" qu'elles "méritaient" de faire partie de la langue française» (citation Assaad 2013 : 9). Et quoiqu'il glose abondamment les emprunts lexématiques, cette modalité de prise en compte du lecteur exogène décroît d'ouvrage en ouvrage, comme si Maalouf imposait les emprunts en langue via son œuvre complète, créant une connivence avec son lectorat fidèle : 87\% sont expliqués dans Le Rocher de Tanios, "50\% dans Les Croisades vues par les Arabes, Léon l'Africain, Samarcande et Les Jardins de lumière, 36\% et $42 \%$ dans Les Échelles du Levant et Le Périple de Baldassare » (Khaled 2013 : 9). La pratique inclusive du glossaire, propice au confort du lecteur exogène, l'invite aussi à entrer, de roman en roman, dans la connaissance du patrimoine linguistique convoqué : Maalouf «insiste sur la notion et la pratique de la réciprocité linguistique et culturelle » (Tsokalidou 2009 : 199), positionnement glottopolitique qu'on peut relier à sa participation au dictionnaire de l'Académie française, emblème du purisme, mais où l'auteur, surnommé par ses collègues «Monsieur l'Orient », projette de compléter de façon approfondie l'approche des étymologies orientales.

Pour éclairer cette visée glottopolitique complexe, situons les positionnements sociolinguistiques de Maalouf dans le contexte des «cent portraits linguistiques » de Gueunier. Connaît-il l'insécurité linguistique ? Sa prise en compte attentive du lecteur exogène le suggèrerait, et sa vérification maniaque de la norme et de l'histoire des mots - lui qui se «lève cinq fois de table au cours d'un même repas pour aller vérifier l'étymologie d'un mot, ou son orthographe exacte» (Maalouf 2004 : 16-17). Son parcours vers la reconnaissance des institutions littéraires les plus centrales, du prix Goncourt à l'Académie française, est-il à interpréter en ce sens ? Toutefois, la lente imposition des emprunts en langue, de moins en moins glosés, et cette capacité à concentrer dans l'espace paradoxal d'un village «microscopique », Kfaryabda, un pan-orient panchronique, vont dans le sens d'une certaine assurance linguistique. Et qu'en est-il des locuteurs libanais ? C'est, «massivement, une attitude d'insécurité linguistique par rapport au français qui se dégage », pour $78 \%$ des 
interrogés (Gueunier 1993: 155). Mais Gueunier souligne que les chrétiens libanais expriment davantage de sécurité linguistique (op. cit. : 57). Elle relève aussi « un amour de la norme classique, traditionnelle, du français ", ainsi qu'une " méfiance générale à l'égard des libanismes, unanimement tenus pour des "écarts" » (op. cit. : 185). Ainsi, les attitudes de Maalouf entrent en convergence avec les locuteurs endogènes sur le thème de la francophilie, mais en divergence sur celui de la variation. Maalouf partage aussi avec la majorité des Libanais des attitudes «massivement favorables au multilinguisme », associées à la promotion de la diversité culturelle et de la paix (op. cit. : 168-170). Les pratiques glossairistiques inclusives de Maalouf et son engagement glottopolitique se dégagent ainsi avec des nuances intéressantes sur la diversité des portraits libanais.

\subsection{Sow Fall : pratique réticente du glossaire}

Dans Le Jujubier du patriarche de Sow Fall, les personnages s'expriment dans un français sénégalisé marqué par les emprunts au wolof. La «mosaïque culturelle» du Sénégal, où interfèrent les civilisations négro-africaine, arabomusulmane et occidentale, est doublée par une forte diversité ethnique et linguistique, avec une vingtaine de groupes linguistiques «éclatés dans une vingtaine, voire une trentaine d'ethnies » (Cissé 2005 : 100-103).

La scénographie des emprunts chez Sow Fall révèle des pratiques plutôt cohérentes. Ses graphies sont souvent conservatrices de la norme sénégalaise, donc proches de la transcription officielle, ainsi pour xalam (« instrument de musique à cordes »), où la lettre $-x$ rend une constrictive vélaire sourde qui n'appartient pas au système phonétique français, souvent rendue par $-k h$ dans les transcriptions suivant la norme de l'alphabet français. Mais on observe un flottement entre les deux logiques sur l'anthroponyme Naaru, également graphié Naarou.

Sur le plan sémantique, on note l'usage d'emprunts dénotant les valeurs, comme wolleré, glosé par «le devoir de sauvegarder les liens, le sens de la fidélité » (Sow Fall 1993: 67). Selon Daff, « la littérature contemporaine [francophone] se fait actuellement l'écho de cette tendance » générale à l'emploi de ce terme wolof dans les usances du français oral (1993 : 276). Pour Lüsebrink, les termes wolofs relevant du réseau lexical des valeurs sont des «sondes emblématiques qui renvoient à une culture, à un système de valeurs et de représentations symboliques » très éloignés de la culture occidentale, si bien que «l'écriture biculturelle de Fall » présuppose «une herméneutique de lecture radicalement différente » (1997 : 28). L'auteure privilégie l'usage des emprunts lexématiques sur leur transposition en français car «la traduction selon les normes d'une bonne traduction les trahit » (Sow Fall $1985: 1)$.

L'analyse quantitative du glossaire sowfallien confirme une pratique réticente de la glose. $\mathrm{Si}$ la mise à distance typographique de l'emprunt, en italiques, rapporte ces formes à la norme du français de référence, en revanche, l'instance qui glose dans le Jujubier du patriarche est reléguée dans la neutralité du paratexte, et la glose dans les marges infrapaginales de l'œuvre; le wolof, langue prêteuse, n'est pas nommé : le narrateur semble conter en connivence avec le lecteur endogène, rendant superflues, dans les gloses très brèves - 0,69 lignes en moyenne -, l'exhaustivité des rubriques, voire la mention de la référence de certains emprunts. En effet, de nos trois auteurs, Sow Fall seule assortit quelquefois l'entrée d'une 
unique rubrique grammaticale, ainsi la glose de waay, limitée au terme « interjection » (1998 : 42) ; la référence de ey (op. cit. : 34) est glosée par «pas de sens particulier ». L'auteure déclare écrire pour «ses concitoyens, pour les lecteurs sénégalais » (citation GiercynsckiBocandé 2005), dans une visée glottopolitique endoréférentielle, confirmée en diachronie : du Revenant à la Grève des Bàttu, seuls «sont mis en glossaire [les termes] dont l'explication semble être indispensable à la compréhension du récit »: il ne s'agit pas d'un «enfermement mais d'un changement de priorité. Le choix, ambigu dans le premier roman, s'est arrêté sans équivoque dans le deuxième, sur un public africain populaire » (Bangura 2000 : 92, citant Minh-Ha).

Cette intégration endoréférentielle du wolof s'avère aussi poétique. Comme Maalouf, Sow Fall pratique les constructions hybrides, ainsi l'expression «baasi salté », dans La Grève des Bàttu, dénotant un couscous... royal. Il s'agit d'un jeu de mots car « l'adjectif qualificatif postposé est un emprunt dialectisé du français qui signifie "sale" », l'auteur jouant sur une paronomase entre les adjectifs français et wolof. Dans la bouche des mendiants, un «Baasi salté buur », « le répugnant couscous du roi », est un retournement savoureux par lequel « la langue s'arroge du coup un pouvoir générateur, non pas de celui du Verbe divin, mais de celui qui permet au pauvre, au dominé [...] de façonner à son tour un réel autre, de faire bouger ensemble le signe et le sens » (Diouf 2009 : 282). Le jeu de détournement des emprunts sert donc une poétique du discours social. Comme chez Maalouf aussi, les titres de romans peuvent comporter des emprunts faisant office de matrices sémantiques. Ainsi, bàttu (« bol, sébile »), indique «une relation de pouvoir [...] et place ceux qui tendent leur bàttu à la merci de la générosité de ceux qui vont y déposer quelque chose ». Or, en français, «au sens propre "battu" signifie "qui reçoit des coups". La première partie du roman de Sow Fall nous montre des mendiants en proie à une répression sanglante ». Et «après avoir été "battus" au sens propre, les mendiants sont "battus" au sens figuré, c'est-à-dire qu'ils ont provisoirement perdu la partie ». Ainsi, Sow Fall «traite de manière métaphorique le problème [...] de tous les groupes sociaux maintenus arbitrairement en état d'infériorité » (Volet 1993 : 73-74). Là encore, au-delà de la mimèsis des langues endogènes, la paronomase génère dans le roman un réseau sémantique et poétique original.

Cette reconfiguration de l'hypotexte des langues endogènes se prolonge dans celle de l'hypotexte épique à la base du Jujubier du patriarche: par la force des choses, « la traduction des chants élégiaques dans L'Appel des arènes est une traduction partielle » (Niang 1998 : 131). Là encore, cette infidélité à l'oralité épique est volontaire, abandonnant « une réception participative pure calquée sur les repères axiologiques que l'épopée véhicule pour une mise à distance [propice au] défigement de la parole stéréotypée » (Valgimigli 2009 : 269). Ce renouvellement serein de la tradition épique par le roman est cohérent avec ces déclarations de l'écrivaine : «dans ma tradition, tout n'est pas transportable à travers le temps [...]. Et à chaque instant de ma vie, ce patrimoine prend de nouvelles formes » (citation Association Vacarme 2001 : 68). De fait, si l'auteure, qui parlait le wolof à la maison et le français à l'école, affirme avoir choisi la langue française parce que les normes écrites du wolof étaient encore mal fixées quand elle a commencé à écrire, elle ajoute que si elle avait « à écrire en wolof, [elle serait] obligée de récréer [sa] propre langue pour l'adapter à [son] travail artistique » (PANAPRESS 2002). 
Ainsi, le parcours de Sow Fall éclaire ses pratiques glossairistiques endoréférentielles et la représentation originale des langues endogènes dans le roman. Etudiante en grammaire et philologie en Sorbonne, enseignante de français au Sénégal, sa trajectoire est marquée par « la tentation autonomiste », portée par un retour à Dakar, dans les années 1970, en pleine « effervescence culturelle, politique et sociale » (Diouf 2009 : 75). Cette tendance aboutit à la fondation d'une maison d'édition sénégalaise, Khoudia, positionnement glottopolitique entreprenant confirmé par son fils, le rappeur Abass Abass, résumant en cette formule l'héritage maternel : «on ne fait pas ce qui marche, on fait marcher ce qu'on fait » (citation Le Gros 2012). Le choix de la langue française n'empêche donc pas l'auteure d'adopter un positionnement nettement endoréférentiel, en indépendance par rapport au regard de l'Occident : « il fallait que la littérature évolue, que nous parlions strictement de nous-mêmes [...] sans éprouver le besoin de nous placer par rapport à l'Europe » (citation HerzbergerFofana 1989 : 101). L'auteure, composant une position paracoloniale plus que postcoloniale, « libérée de toute référence hiérarchique et insensible à toute insécurisation », échappe ainsi au carré glottopolitique de Caitucoli qui situait les attitudes des écrivains francophones en référence à la centralité $(2004 \mathrm{~b}: 17)$. Toutefois, la pratique sowfallienne du français reste normativiste - la langue d'écriture, dialogues mis à part, étant globalement, comme chez Maalouf, assez classique - ce que confirment ses déclarations. Si « le style de l'écrivain, c'est ce qui le distingue [...], ce qui fait qu'à travers le français qu'il utilise, on devine [...] son âme et l'âme du peuple qu'il représente », toutefois, «ces pratiques langagières ne sont pas incompatibles avec l'usage d'une langue correcte »: il ne s'agit donc pas de «parler nos langues en français. Ce serait une double trahison vis-à-vis de nos langues et du français. Et cette trahison comporte des risques car tout le monde n'est pas Ahmadou Kourouma » (Sow Fall 1985 : 3). La comparaison explicite à Kourouma en témoigne, la pratique endoréférentielle du glossaire sowfallien n'implique pas une volonté de subversion de la langue.

Ces choix sont-ils cohérents avec les positionnements sociolinguistiques des locuteurs sénégalais? Les langues locales sont créditées dans leur discours épilinguistique « de vertus participant de l'affirmation de l'identité africaine dans un contexte où le français étend de plus en plus son rayonnement» (Mbodj 2005); la promotion sowfallienne des valeurs sénégalaises par le langage s'inscrit dans un continuum avec les attitudes générales. La valorisation de la norme centrale par l'auteure est aussi compatible avec les pratiques endogènes dominantes du français, « résolument marquées par des fonctions académiques et emblématiques qui mettent l'accent sur des finalités ostentatoires expliquant la recherche constante du "beau parler", du "parler bien" » (Ndao 2002 : 61). Les enquêtes confirment en outre l'affirmation souvent sereine d'une identité multiple, sénégalaise, wolofe, francophone : «les informateurs construisent trois types d'identités, notamment ceux qui se basent sur les paramètres ethnique, spatial et macroculturel. La plupart du temps ces types d'identités sont présents simultanément dans [leur] imaginaire » (Versluys 2010 : 260). Sow Fall se démarque davantage par sa « revendication d'une norme endogène », via le traitement endoréférentiel des emprunts : celle-ci «apparaît faiblement dans les réponses » des interrogés (Ndao 2002 : 58) ; mais il faut nuancer ces éléments, en soulignant «l'ambiguïté des attitudes des Sénégalais vis-à-vis de la langue française », « soupçonnée de véhiculer des valeurs étrangères », mais qu'on « accepte pour son utilité présente, à condition qu'elle porte des marques de sénégalité »(op. cit. : 58-59 citant Ndiaye-Corréard). Ce positionnement diglossique ambigu suggère que « le concept d'appropriation ne rend pas tout à fait compte de 
la réalité de la situation ou des sentiments étant donné le caractère non achevé de son assomption et la revendication concomitante d'une position privilégiée dans la pratique d'un français normatif voire "surnormatif" ». Ainsi, «les locuteurs dévalorisent les normes africaines tout en les préférant à la norme centrale [...]; phénomène propre au fonctionnement diglossique qui établit un double mouvement (de dévalorisation/appropriation) » (op. cit. : 59).

\subsection{Kourouma : pratique proliférante du glossaire}

Chez l'auteur ivoirien, la langue française est tropicalisée par le malinké, adjectif mandé désignant « un dialecte du nord de la Côte d'Ivoire (chez Kourouma c'est la forme sublimée des différentes langues mandé) » (Prignitz 2005 : 51). Ce pays frontalier de plusieurs États Libéria, Guinée, Mali, Burkina Faso et Ghana - est marqué par une forte hétérogénéité linguistique, avec une soixantaine de langues ivoiriennes, dont la multiplicité est à corréler à celle des ethnies. Le français, seule langue à s'être imposée comme véhiculaire, connaît un phénomène de forte appropriation sous les espèces notamment du Français populaire ivoirien (FPI), « le français de l'Ivoirien modeste, celui sur qui l'école a eu peu ou pas d'influence » (Barry 2007 : 33).

Pour Kourouma, si le français offre un accès privilégié à l'édition, les impératifs romanesques exigaient sa malinkisation pour «donner aux personnages leur langage naturel » (citation Chanda 2003). La visée mimétique engage ainsi une riche nomenclature d'emprunts lexématiques, engendrant aussi de nombreux calques sémantiques. Mais la visée de Kourouma est plus complexe. Si on rencontre de multiples interjections connotatrices d'oralité, tel l'omniprésent «Walahé ! » («au nom d'Allah»), leur répétition obsessionnelle, assortie d'une glose systématique, décrédibilise l'imitation de l'oral au profit d'une esthétique burlesque. Les calques font aussi entendre, dans le français, des expressions endogènes, mais non sans manipulations de l'auteur : dans l'expression « bon pied la route », qui devient une locution verbale, accompagnée tantôt de l'auxiliaire être, tantôt d'avoir (« elle était partie pied la route en Sierra Leone»(Kourouma $2000: 168$ ), « ils ont pris pied la route, le chemin du Nord» (op. cit. : 135), voire « nous avons pris notre pied la route pour quitter Zorzor » (op. cit. : 87 ), le défigement causé par la collusion cocasse entre «prendre la route » et «prendre son pied » active une polysémie inédite.

Comme nos autres auteurs, Kourouma souligne la valeur emblématique de l'emprunt, lexématique ou sémantique, par sa présence en titre : monnè, «outrages et défis »; les soleils « des Indépendances », leur conférant là aussi la valeur de matrice sémantique, qui tout à la fois double la visée mimétique d'une visée poétique, et l'obscurcit. Kouassi relève la variabilité des sens de soleil, signifiant tantôt «la journée », tantôt «l'équivalent de l'année » (2007 : 123). Soleil est aussi pris en son sens français, si bien qu' «à partir du stimulus "soleil" » se réalise une prolifération des sens grâce à l'oscillation sans cesse créatrice entre le dit et le non-dit, entre expériences antérieures des utilisateurs fictionnels (personnages, narrateurs, focalisateurs) et [celles] de divers lecteurs, enfin entre sens dénotés supposés fixes et le champ illimité de connotations et de devenir » (Asaah 2006 : 2). La polysémie de la lexie est activée jusqu'à en faire un hybride : pour Diandue, les emprunts sont « des condensés idéologiques et esthétiques propres à figurer l'identité culturelle malinké 
confinée dans la langue malinké que Kourouma utilise pour créer un discours romanesque particulier », partant, « des traits particuliers de [son] esthétique » (2013: 78).

Cet usage infidèle des lexies françaises et malinkées suscite la perplexité d'Edema, gêné par la pratique du défigement et cette polysémie forcée peu représentatives de l'usage : pour lui, Kourouma «oublie VOLONTAIREMENT que le sens entier de l'expression ne résulte pas de la somme des sens de ses éléments constitutifs »; « les catachrèses [...] sont réactualisées en traduction », devenant « des mots de jeu [sic] que le lecteur se doit de découvrir » (2004 : 239-240). Seule la visée mimétique semble légitime au critique, oublieux du travail poétique et glottopolitique de l'écrivain. Mais rares sont ceux qui peuvent juger de cette scénographie qui non seulement tropicalise la langue française, mais, Sow Fall l'affirmait, amène à recréer aussi les langues endogènes. Pour Moudileno, il est impératif «d'avoir des critiques conversant dans les langues africaines », car pour «rendre justice au travail des écrivains africains qui œuvrent dans la langue française », il faut « rendre compte de manière détaillée des jeux de transcription, récupération, manipulation auxquels ceux-ci se livrent, empruntant tantôt à une langue maternelle qu'ils pratiquent à l'oral, tantôt aux inventions de la rue, tantôt à leur imagination uniquement » (2003: 66-67).

L'analyse statistique du glossaire d'Allah n'est pas obligé révèle une pratique proliférante de la glose. Comme les significations polysémiques, les définitions y prolifèrent. Traducteur universel, Birahima, par l'absence d'italiques et la réciprocité des définitions, brouille les normes dans un traitement polyréférentiel de l'emprunt en répondant entre parenthèses aux lacunes linguistiques des narrataires exogènes et endogènes, avec une longueur moyenne de 1,3 lignes par mot, qui situe le glossaire kouroumien à l'intermédiaire entre les pratiques de Sow Fall et Maalouf. L'incipit du roman donne le ton, qui s'ouvre sur des remarques différentielles. Birahima, narrateur pluristyle, glose pour tous : « des toubabs (toubab signifie blanc) colons, des noirs indigènes sauvages d'Afrique et des francophones de tout gabarit » (Kourouma $2000: 11$ ), le français standard comme la variation régionale.

Simple jeu littéraire? Les déclarations de Kourouma confirment cette pratique polyréférentielle de la glose, avec une construction des lectorats équilibrée : il a «privilégié le lecteur européen [...]. Mais le lecteur africain est quand même un destinataire privilégié » (citation Caitucoli 2004b : 17). La nomenclature du glossaire rappelle le panorientalisme de Maalouf, le recours massif aux africanismes locaux et régionaux, voire issus « de l'ensemble du continent », suggérant la recherche d'une «dimension véritablement panafricaine » (Mathieu-Job 2003 : 150). Les attitudes épilinguistiques déclarées de Kourouma entrent aussi en cohérence avec ses pratiques: l'auteur argue de son parcours pour expliquer son audacieuse tropicalisation de la langue française - « je ne suis pas littéraire mais mathématicien ; aussi me suis-je toujours senti libre, tranquille et à l'aise vis-à-vis du français », sans «peur de transgresser » (cité par Caitucoli 2004b : 23). Il affiche revanche une légère insécurité linguistique à l'égard du malinké : «mon long exil m'a fait perdre un peu la langue malinkée, et actuellement je pense en français » (citation Fénoli 2004).

Certains de ces points convergent avec les attitudes épilinguistiques d'une partie des Ivoiriens, ressentant « le conflit individuel qu'implique l'adoption d'une langue seconde [...]. Un enquêté déclare le plurilinguisme responsable de la déculturation : "Il m'a empêché d'avoir une maîtrise parfaite de ma propre langue. Je me rends compte [...] que je connais 
encore mal les arcanes de la pensée mythique, religieuse, l'organisation et la vie culturelle de mon propre milieu" »(Ploog 2001 : 431). En outre, les Ivoiriens ont conscience « du caractère spécifique de "leur français" qu'ils ne stigmatisent pas outre mesure »; un interrogé décrit le FPI comme « le français du peuple », que «tout Ivoirien [...] comprend sans même l'apprendre », qui «nous identifie et brise les barrières tribales et les particularismes » (Kouadio N'Guessan 2008 : 190). Une langue qui réunit et qu'on peut s'approprier, c'est bien celle portée par les pratiques glossairistiques de Kourouma. Précédant en cela le roman kouroumien, La chronique de Moussa et la bande dessinée Dago avaient commencé à donner au FPI la légitimité de la «chose écrite», et une «légitimité [...] lexicographique », en proposant un paratexte de notes «précisant les usages des mots ou expressions nouvellement utilisés » (Mel Gnamba \& Kouadio N'Guessan 1990 : 51). Notre mise en perspective des pratiques d'écriture de Kourouma n'est pas sans intérêt pour contextualiser son œuvre : génial démiurge d'une œuvre fondatrice pour les littératures francophones, en rupture avec la tradition, il a aussi su s'inscrire avec bonheur dans la continuité de certaines pratiques et représentations endogènes.

\section{Conclusion}

Comment aborder les enjeux des représentations des langues dans les romans hétérolingues? La prise en compte du traitement des langues endogènes via l'analyse des pratiques glossairistiques pourrait permettre de dépasser les antinomies de la mimésis posées en partie théorique. Si la critique des œuvres hétérolingues ne cherche dans ces textes que des faits de langue, privilégiant une visée mimétique, elle occulte la portée poétique de l'œuvre. En effet, quoique «la relation du texte à l'Afrique [soit] déterminante», «l'ancrage est devenu encrage et, en cela, a perdu de sa transparence: la «double allégeance de l'écriture littéraire [...] "porte à la fois l'aliénation de l'Histoire et le rêve de l'Histoire"». Pour Boudreault, la pratique romanesque de Kourouma figure cette «zone transactionnelle où les mots se trouvent constamment engagés, passant d'une temporalité à une autre » (2006 : 96, citant Barthes), dans une dynamique proprement glottopolitique du langage. Inversement, si elle fond les langues convoquées par le roman dans l'indistinct effet de l'outre-langue, la critique ignore l'art auctorial de revitaliser et défiger toute langue, ce rapport étrange, fait de continui et de ruptures, entre langue commune et langue littéraire, dont la formule - vraie de tout écrivain, français ou francophone - pourrait être : français ou endogènes, «ce sont les mots de tous les jours, et ce ne sont point les mêmes » (Claudel 1950:117), tant il est vrai que le jeu de mots et le jeu des mots se trouve, toujours, en langue aussi bien qu'en littérature.

Par l'analyse écologique de la visée glottopolitique du glossaire romanesque, dont la portée a été envisagée en ses points de convergence et de divergence avec les positionnements épilinguistiques endogènes, cette étude souhaitait réinscrire les démarches des écrivains dans leur rapport non seulement avec la centralité, mais aussi avec les champs endogènes émergeants. Il s'agirait en définitive d'aller au-delà de l'insistance sur ce qui semble une même « condition diglossique », motif commun aux critiques des littératures francophones, et de s'assurer la «collaboration de linguistes, de critiques au fait des langues africaines et d'Occidentaux sachant dépasser l'émerveillement de l'étrangeté de la langue », afin de réaliser des «analyses rigoureuses sur la créativité langagière des écrivains d'aujourd'hui » (Moudileno 2003 : 67). Kane regrette que souvent, la démarche critique souligne soit « une 
spécificité » de tel ou tel terroir francophone, soit « une continuité en regard de la littérature française », sans vraiment articuler ces points de vue. Reste, pour ce faire, à instaurer « un nouveau débat, en identifiant les paramètres de l'histoire : nécessité d'une délimitation de la littérature africaine en littératures nationales ou régionales ; recours à une méthodologie rigoureuse qui prend en ligne de compte la contextualisation $»$ : de ce point de vue, « l'histoire littéraire [...] francophone est encore à écrire » (1991 : 9).

Mais parallèlement, la mimèsis de l'oral dans le roman, qu'elle soit véhiculée par l'embrayeur de paratopie qu'est l'emprunt, ou bien, en littérature hexagonale, par la textualisation de stéréotypes du langage oral, dévoile aussi la solidarité profonde des dynamiques des histoires littéraires, qui pourraient s'écrire au singulier et non dans une binarité. L'évolution des littératures vers un «devenir-discours », portée par Céline ou par Kourouma, semble manifester une même dynamique : en France, « autonomisée, mettant en place des formes et des lois propres peu à peu figées comme marqueurs sémiotiques de littérarité, la langue littéraire aurait [...] paradoxalement fini par rejoindre l'idiome » (Piat 2012 : 5). Parallélisme frappant: c'est «en tant qu'authentiques représentants d'une civilisation de l'oralité que les écrivains africains d'expression française ont produit la littérature la plus conforme aux normes écrites conventionnelles »; à l'inverse, «c'est quand ils se sont intégrés davantage à une civilisation de l'écrit imprimé qu'ils ont eu les moyens d'opérer la mise en oralité de cette littérature»(Derive 2004 : 196). Perspective plus endogène pour examiner la singularité des trajectoires à partir de champs spécifiques ; perspective plus unitaire pour découvrir les mouvements dialectiques profonds à l'œuvre dans la littérarité : tels sont les horizons que dévoile une analyse attentive des glossaires hétérolingues.

\section{Références}

Alami, A. O. (2005). L'épreuve de la voix : la narration face au personnage dans le roman arabe francophone. Le cas du Rocher de Tanios d'Amin Maalouf. Horizons Maghrébins - Le droit à la mémoire, 52, 31-45.

Arrivé, M. (1969). Postulats pour la description linguistique des textes littéraires. Langue française, 3 , 3-13.

Asaah, A. H. (2006). Le soleil entre fixité et devenir : le lecteur devant la présence solaire énigmatique dans Les Soleils des Indépendances d'Ahmadou Kourouma. Éthiopiques, 76. En ligne :

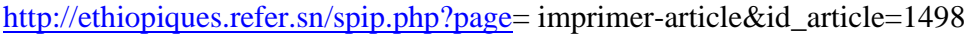

Assaad, N. (2004). Une mutation linguistique : le cas d'Amin Maalouf. Cahiers de l'AIEF, 56, 457-483.

Assaad, N. (2013, octobre). Le Rocher de Tanios : une osmose langagière. Communication présentée à la journée Amin Maalouf du Centre Supérieur de la Recherche, Université de Saint-Esprit de Kasilik, USEK, Juniyah. En ligne : http://www.usek.edu.lb/CSR/Annonces.Journee_Amin_Maalouf

Association Vacarme (2001). Ecrivain-fleuve. Entretien avec Aminata Sow Fall. Vacarme, 3 (16), $67-$ 68.

Bakhtine, M., Esthétique et théorie du roman, Gallimard, Paris, 1978. 
Bangura, A. S. (2000). Islam and the West-african Novel : the politics of representation. London \& Boulder : Lynne Rienner Publishers.

Barry, A. O. (2007). Pour une sémiotique trans-culturelle de l'écriture littéraire francophone d'Afrique. Synergies, 2, 19-39.

Blanchet, P. (2007). Quels « linguistes » parlent de quoi, à qui, quand, comment et pourquoi ? Pour un débat épistémologique sur l'étude des phénomènes linguistiques. In P. Blanchet, L.-J. Calvet \& D. De Robillard (Dirs.), Un siècle après le Cours de Saussure : la linguistique en question. Carnets d'Atelier de Sociolinguistique, 1 (pp. 229-294). Paris : L'Harmattan.

Boudreault, L. (2006). L'ceuvre romanesque d'Ahmadou Kourouma et sa critique. Mémoire de maîtrise en études littéraires, Université Laval, Québec.

Caitucoli, C. (2004a). Présentation. Glottopol, 3, 2-5.

Caitucoli, C. (2004b). L'écrivain africain francophone agent glottopolitique : l'exemple d'Ahmadou Kourouma. Glottopol, 3, 6-25.

Calvet, L.-J. (1999). Pour une écologie des langues du monde. Paris : Plon.

Chanda, T. (2003). Les derniers mots d'Ahmadou Kourouma. En ligne sur le site de RFI Afrique : http://www1.rfi.fr/actufr/articles/048/article_25500.asp

Cissé, M. (2005). Langues, État et société au Sénégal. SudLangues, 5, 99-133. En ligne : http://www.sudlangues.sn/

Claudel, P. (1950 [1910]). Cinq grandes odes. Paris : Gallimard.

Daff, M. (1993). Relevé de quelques particularités lexicales du français au Sénégal à partir d'un corpus de textes écrits : degré d'intégration de ces particularités. In D. Latin, A. Queffélec \& J. Tabi-Manga (Eds.), Inventaire des usages de la francophonie : nomenclatures et méthodologies (pp. 275-284). Paris : John Libbey Eurotext.

Dakroub, F. (2011). La voix «non-visible» du truchement. Etude de l'hétérolinguisme dans le texte romanesque d'Amin Maalouf. Les cahiers du GRECELF, 2, 261-279.

Dakroub, F. (2014). Les Roum chez Amin Maalouf : des Castillans ou des Byzantins ? En ligne sur le site L'Orient d'Amin Maalouf : http://amaalouf.hypotheses.org/349\#more-349

De Pietro, J.-F. (1988). Vers une typologie des situations de contacts linguistiques. Langage et Société, $43,65-89$

Derive, J. (2004). Imitation et transgression. De quelques relations entre entre la littérature orale et et la littérature écrite dans les cultures occidentales et africaines. Cahiers de Littérature orale, 56, $175-$ 200.

Deroy, L. (1956). L'Emprunt linguistique. Paris : Les Belles Lettres.

Diouf, M. (2009). L'énonciation de l'exil et de la mémoire dans le roman féminin francophone : Anne Hébert, Aminata Sow Fall, Marguerite Duras. Mémoire de doctorat en Etudes littéraires, Université Laval, Québec.

Diandue, P. (2013). Réflexions géocritiques sur l'œuvre d'Ahmadou Kourouma. Paris : Publibook.

Dufour, P. (2004). La Pensée romanesque du langage. Paris : Seuil.

Edema, A. B. (2004). Les xénismes dans les romans africains : entre citations, traduction et créativité lexicale. Le Français en Afrique. Revue du Réseau des Observatoires du Français contemporain en Afrique, 19, 226-243. 
Favart, F. (2010). La représentation de l'oralité populaire dans quelques romans du second $20^{\mathrm{e}}$ siècle (1966-2006). Langage et société, 1 (131), 147-148.

Fénoli, M. (2004). Les « contre-dires » de l'histoire. Notre Librairie, Revue des littératures du Sud, 155$156,77-81$.

Fonseca, F. I. (1996). Quand dire c'est feindre : théorie linguistique et fiction littéraire. In N. Faria (Ed.), Language and Literature Today. Proceedings of the XIXth Triennial Congress of the International Federation for Modern Languages and Literatures (Brasília, August 1993), vol. II (pp. 880-885). Brasília : Universidade de Brasília.

Frey, C. (2008). Regards de locuteurs francophones sur la diversité lexicale en Afrique. Représentations, identités, intercompréhension. In Actes du colloque : La langue française dans sa diversité (pp. 17-38). Québec : Direction des Relations publiques du ministère de la Culture, des Communications et de la Condition féminine.

Gauvin, L. (1999). Faits et effets de langue : le réalisme comme désir. In L. Gauvin (Dir.), Les langues du roman : du plurilinguisme comme stratégie textuelle (pp. 53-72). Montréal : P.U.M.

Gauvin, L. (2001). Une situation d'interlangue : les romans d'Axel Gauvin. In K. R. Issur \& V. Y. Hookoomsing (Dirs.), L'Océan Indien dans les littératures francophones. Pays réels, pays rêvés, pays révélés (pp. 153-166). Paris : Karthala.

Gauvin, L. (2008). Décalage langagier : le sentiment de la langue chez les écrivains québécois. In M. Ngalasso-Mwatha (Dir.), Linguistique et poétique. L'énonciation littéraire francophone (pp. 15-22). Bordeaux : P.U.B.

Giercynscki-Bocandé, U. (2005). La Femme et les lettres. Aminata Sow Fall et l'avenir de la femme au Sénégal, In Aminata Sow Fall : une femme de lettres de dimension internationale, Colloque du 08 au 10 avril 2005.

Philippe, G. \& Piat, J. (Dir.). (2009). La langue littéraire : une histoire de la prose en France de Gustave Flaubert à Claude Simon. Paris : Fayard.

Grutman, R. (1996). Langues étrangères et savoir romantique : considérations préliminaires. TTR, 9 (1), 71-90.

Grutman, R. (1997). Des langues qui résonnent. L'hétérolinguisme au XIX siècle québécois. Québec : Fides-CETUQ.

Grutman, R. (2002). Les motivations de l'hétérolinguisme : réalisme, composition esthétique. In F. Brugnolo \& V. Orioles (Dirs), Eteroglossia e plurilinguismo letterario, vol. 2 : plurilinguismo e letteratura (pp. 291-392). Rome: Il Calamo - Centro internazionale sul plurilinguismo dell'Università degli Studi di Udine.

Grutman, R. (2005). La textualisation de la diglossie dans les littératures francophones. In J. Morency, H. Destrempes, D. Merkle \& M. Pâquet (Éds), Des cultures en contact : visions de l'Amérique du Nord francophone (pp. 201-223). Québec : Nota bene.

Guespin L. (1985). Introduction. Matériaux pour une glottopolitique. Cahiers de linguistique sociale, 7 , 14-32.

Gueunier, N. (1993). Le français du Liban : cent portraits linguistiques. Paris : Didier-Érudition.

Herzberger-Fofana, P. (1989). Écrivains africains et identités culturelles. Entretiens. Tübingen : Stauffenburg.

Kanaan, L. (2011). Reformulations, contacts de langues et compétence de communication : analyse linguistique et interactionnelle dans des discussions entre jeunes Libanais francophones. Mémoire de doctorat en Sciences du langage, Université d'Orléans, Orléans. 
Kane, M. (1991). Sur l'histoire littéraire de l'Afrique subsaharienne francophone. Etudes littéraires, 24 (2), 9-28.

Khaled, M. (2013, octobre). Le métissage linguistique dans les romans d'Amin Maalouf. Communication présentée à la journée Amin Maalouf du Centre Supérieur de la Recherche, Université de Saint-Esprit de Kasilik, USEK, Juniyah. En ligne : http://www.usek.edu.lb/CSR/Annonces.Journee_Amin_Maalouf

Kouadio N'Guessan, J. (2008). Le français en Côte d'Ivoire : de l'imposition à l'appropriation décomplexée d'une langue étrangère. Documents pour l'histoire du français langue étrangère ou seconde, 40-41, 179-197.

Kouassi, G. (2007). Le phénomène de l'appropriation linguistique et esthétique en littérature africaine de langue française. Le cas des écrivains ivoiriens : Dadié, Kourouma et Adiaffi. Paris : Publibook.

Latin, D. (2006). Corpus littéraire et corpus linguistique : une solidarité nécessaire à la description de l'"africanité" du français. In Appropriation de la langue française dans les littératures francophones de l'Afrique subsaharienne, du Maghreb et de l'Océan Indien: Actes des journées scientifiques des réseaux de chercheurs concernant la langue et la littérature (pp. 143-150). Paris : AUF.

Le Gros, J. (2012). Le palais appartient au peuple. Les politiques sont juste des locataires. En ligne sur le site Africultures : http://www.africultures.com/php/?nav=article\&no=10712

Lüsebrink, H.-J. (1997). Domination culturelle et paroles résistantes. De la dimension conflictuelle dans la communication interculturelle. In F. Tétu de Labsade (Ed.), Littérature et dialogue interculturel (pp. 19-32). Laval : P.U.L.

Maalouf, A. (2004). Origines. Paris : Grasset \& Fasquelle.

Maingueneau, D. (2004) : Le discours littéraire. Paratopie et scène d'énonciation. Paris : Armand Colin.

Mathieu-Job, M. (2003). L'intertexte à l'œuvre dans les littératures francophones. Pessac : P.U.B.

Mbodj, C. (2003, septembre). Coexistence dynamique du français et des langues partenaires au Sénégal. Didactique et aménagement linguistique en Afrique francophone. Communication présentée à la $\mathrm{XX}^{\mathrm{e}}$ Biennale de la langue française, La Rochelle. En ligne http://www.biennalelf.org/b20/

Mel Gnamba, B. \& Kouadio N'Guessan, J. (1990). Variétés lexicales du français en Côte d'Ivoire. In A. Clas \& B. Ouoba (Dirs..), Visages du français. Variétés lexicales de l'espace francophone (pp. 5158). Paris : AUPELF-UREF / John Libbey Eurotext.

Meizoz, J. (2001). L'âge du roman parlant. Ecrivains, critiques, linguistes et pédagogues en débat. Genève : Droz.

Moudileno, L. (2003). Littératures africaines francophones des années 1980 et 1990. Dakar : CODESRIA.

Ndao, P. A. (2002). Le français au Sénégal : une approche polynomique. SudLangues, 16, 51-64. En ligne : http://www.sudlangues.sn/

Niang, S. (1998). Modalités de la signification littéraire chez Mariama Bâ et Aminata Sow Fall. In E. Makward, T. Ravell-Pinto \& A. Songolo (Eds.), The growth of African literature. Twenty-five years after Dakar and Fourah Bay (pp. 123-133). Trenton NJ : Africa World Press.

PANAPRESS (2002). Aminata Sow Fall annonce la sortie de son prochain roman. En ligne : http://www.panapress.com/Aminata-Sow-Fall-annonce-la-sortie-de-son-prochain-roman--13607744-17-lang4-index.html 
Piat, J. (2012). Que reste-t-il de la langue «littéraire»? Revue critique de fixxion française contemporaine, 541, 5-13. En ligne : http://www.revue-critique-de-fixxion-francaisecontemporaine.org/rcffc/article/view/fx03.02/541

Ploog, K. (2001). Le non standard entre norme endogène et fantasme d'unicité. L'épopée abidjanaise et sa polémique intrinsèque. Cahiers d'études africaines, 163-164, 423-442.

Pöll, B. (2005). Le français langue pluricentrique ? Études sur la variation diatopique d'une langue standard. Francfort-sur-Main/Berlin/Bern : Lang.

Prignitz, G. (2004). Récupération et subversion du français dans la littérature contemporaine d'Afrique francophone : quelques exemples. Glottopol, 3, 26-43. En ligne http://www.univrouen.fr/dyalang/glottopol/numero_3.html

Prignitz, G. (2005). Les visages de la culture dans l'œuvre de Kourouma. Dialogos, 11, 51-60.

Queffélec, A. (1998). Des migrants en quête d'intégration : les emprunts dans les français d'Afrique. Le Français en Afrique, 12, 245-256.

Schwob, D. (2014). Personnages en quête de référence(s) dans trois romans hétérolingues en langue française : pour une poétique comparée de la glose. Fabula-LHT, 11. En ligne : http://www.fabula.org/lht/12/schwob.html

Solon, P. (2004). Ecrire l'interculturalité : l'exemple de l'écrivain francophone Amin Maalouf. In H.-J. Lüsebrink \& K. Städtler (Eds.), Les Littératures africaines de langue française à l'époque de la postmodernité : État des lieux et perspectives de la recherche (pp. 163-177). Oberhausen : Athena.

Sow Fall, A. (1985). Pratiques langagières dans la littérature négro-africaine de langue française. Éthiopiques, 40-41. En ligne : http://ethiopiques.refer.sn/spip.php?article986

Thibault, A. (2006). Glossairistique et littérature francophone. Revue de linguistique romane, 277-78, 143-179.

Tsokalidou, R. (2009). Questions de langue et d'identité : le cas d'Amin Maalouf. Synergies, 2, 195-202.

Valgimigli, N. (2009). Non-dit et parole oblique féminine dans quelques romans francophones. In C. Andriot-Saillant (Dir.), Paroles, langues et silences en héritage. Essais sur la transmission intergénérationnelle aux $X X^{e}$ et XXI siècles (pp. 259-278). Clermont-Ferrand : P.U. Blaise Pascal.

Versluys, E. (2010). Langues et identités au Sénégal. Paris : L'Harmattan.

Volet, J.-M. (1993). La parole aux Africaines ou l'idée de pouvoir chez les romancières d'expression française de l'Afrique sub-saharienne. Amsterdam : Rodopi.

Volterrani, E. (2001). Amin Maalouf : entretien à deux voix. En ligne sur le site officiel d'Amin Maalouf : http://www.aminmaalouf.org/

Wissner, I. (2010). Les diatopismes du français en Vendée et leur utilisation dans la littérature : l'œuvre contemporaine d'Yves Viollier. Thèse de Doctorat en philosophie, Université Paris-Sorbonne, Philosophische Fakultät der Universität zu Bonn. 